\title{
Genomic characterisation of the new Dickeya fangzhongdai species regrouping plant pathogens and environmental isolates
}

Špela Alič ${ }^{\varkappa 2}$, Jacques Pédron $^{3}$, Tanja Dreo ${ }^{1}$ and Frédérique Van Gijsegem ${ }^{3^{*}}$ (D)

\begin{abstract}
Background: The Dickeya genus is part of the Pectobacteriaceae family that is included in the newly described enterobacterales order. It comprises a group of aggressive soft rot pathogens with wide geographic distribution and host range. Among them, the new Dickeya fangzhongdai species groups causative agents of maceration-associated diseases that impact a wide variety of crops and ornamentals. It affects mainly monocot plants, but $D$. fangzhongdai strains have also been isolated from pear trees and water sources. Here, we analysed which genetic novelty exists in this new species, what are the D. fangzhongdai-specific traits and what is the intra-specific diversity.

Results: The genomes of eight $D$. fangzhongdai strains isolated from diverse environments were compared to 31 genomes of strains belonging to other Dickeya species. The $D$. fangzhongdai core genome regroups approximately 3500 common genes, including most genes that encode virulence factors and regulators characterised in the $D$. dadantii 3937 model strain. Only 38 genes are present in D. fangzhongdai and absent in all other Dickeyas. One of them encodes a pectate lyase of the PL10 family of polysaccharide lyases that is found only in a few bacteria from the plant environment, soil or human gut. Other D. fangzhongdai-specific genes with a known or predicted function are involved in regulation or metabolism.

The intra-species diversity analysis revealed that seven of the studied $D$. fangzhongdai strains were grouped into two distinct clades. Each clade possesses a pool of 100-150 genes that are shared by the clade members, but absent from the other $D$. fangzhongdai strains and several of these genes are clustered into genomic regions. At the strain level, diversity resides mainly in the arsenal of T5SS- and T6SS-related toxin-antitoxin systems and in secondary metabolite biogenesis pathways.

Conclusion: This study identified the genome-specific traits of the new D. fangzhongdai species and highlighted the intra-species diversity of this species. This diversity encompasses secondary metabolites biosynthetic pathways and toxins or the repertoire of genes of extrachromosomal origin. We however didn't find any relationship between gene content and phenotypic differences or sharing of environmental habitats.
\end{abstract}

Keywords: T5SS, T6SS, NRPS/PKS, Zeamine, oocydin A, Plant-bacteria interactions, Plasmid, Dickeya fangzhongdai

\footnotetext{
* Correspondence: vangijse@agroparistech.fr

${ }^{3}$ Institute of Ecology and Environmental Sciences of Paris, Sorbonne

Universités, UPMC Univ Paris 06, Diderot Univ Paris 07, UPEC Univ Paris 12,

CNRS, INRA, IRD, 4 Place Jussieu, 75005 Paris, France

Full list of author information is available at the end of the article
}

(c) The Author(s). 2019 Open Access This article is distributed under the terms of the Creative Commons Attribution 4.0 International License (http://creativecommons.org/licenses/by/4.0/), which permits unrestricted use, distribution, and reproduction in any medium, provided you give appropriate credit to the original author(s) and the source, provide a link to the Creative Commons license, and indicate if changes were made. The Creative Commons Public Domain Dedication waiver (http://creativecommons.org/publicdomain/zero/1.0/) applies to the data made available in this article, unless otherwise stated. 


\section{Background}

Soft rot Pectobacteriaceae are Enterobacterales responsible for considerable economic losses in several important crops and ornamental plants [1-3]. Their virulence is mainly due to the production and secretion of a battery of plant cell wall degrading enzymes (PCWDEs) that cause maceration of the plant tissue; however, several other virulence factors have also been characterized [2, 4]. These bacteria often exhibit a very broad host range, and recent outbreaks in potato, for example, resulted from the action of a cohort of bacteria belonging to different Pectobacteriaceae species in a complex population dynamics history [5]. The Pectobacteriaceae family includes two genera comprising soft rot bacteria, Pectobacterium and Dickeya. The Dickeya genus was formed in 2005 by the reclassification of former Erwinia chrysanthemi into six species [6]. It has recently undergone multiple phylogenetic changes, including the addition of three new species, Dickeya solani [7], Dickeya aquatica [8] and, more recently, Dickeya fangzhongdai [9].

The description of this last new species was based on three isolates from pear trees in China with bleeding canker necrosis [9], but it was extended by a large number of strains isolated from monocot plants from Japan $[10,11]$. D. fangzhongdai strains were associated with soft rot symptoms of many ornamental and economically important staple food plants $[10,12,13]$, thereby highlighting the broad host range of the species.

While there is little information regarding associated economic damages and the extent of its occurrence in different host plants outside of Asia, Alič et al. [14] recently identified $D$. fangzhongdai as the causative agent of soft rot of orchids in commercial production in Europe, starting with material from Asia [11]. Moreover, as previously reported, bacteriophages of different families, and active against $D$. fangzhongdai, were isolated from a wastewater treatment plant not associated to the orchid production site. This would suggest that $D$. fangzhongdai bacteria may be more widespread in nature than could currently be concluded on the basis of symptoms in plants. Its occurrence in water would suggest that it may potentially have a wider ecological niche than genomically close Dickeya spp., that is, Dickeya dadantii, Dickeya dianthicola, and D. solani.

Previous experience with $D$. solani has shown that novel species or isolates can lead to clonal spread and high losses in affected host plants [15]. Together with repeated introductions of $D$. fangzhongdai, the co-occurrence of genetically and phenotypically diverse strains on the same plants (e.g., B16 and S1 on orchids, as reported by Alič et al. [11]) increases the probability of the development of recombined strains with novel pathogenic potential and may present a risk to agriculturally important plants. Their aggressiveness, high maceration potential on various plant tissues, and persistence in potato plants further exacerbate the risk for agriculture.

Therefore, in this paper, we analysed the genomic characteristics of the D. fangzhongdai species, compared it to the other Dickeya species and determine the interand intra- species diversity. The study addressed the question whether the presence of the isolates in a specific environment is associated to a specific set of genes (water vs plant symptoms, monocots vs dicots, different geographical origin). We also analysed the virulence gene arsenal, in order to evaluate the virulence potential of this species.

\section{Methods \\ Dickeya strain selection}

All $D$. fangzhongdai genomes publicly available in the NCBI database were included in this study. These genomes were compared to five $D$. solani, four $D$. dadantii, five $D$. dianthicola, five $D$. chrysanthemi, seven $D$. zeae, one D. aquatica, two D. paradisiaca and two unassigned Dickeya genomes extracted from the NCBI database. Information on the provenance and genomic data of the $D$. fangzhongdai strains used in this study are summarized in Table 1. The accession numbers and phylogenetic position of the other Dickeya strains used for the SiLix analyses are presented in Additional file 1: Figure S1.

\section{Genome sequencing and assembly}

The D. fangzhongdai B16 and S1 draft genomes assembled from Ion Torrent sequencing data [16] exhibited poor quality. Therefore, both genome sequences were improved by Illumina sequencing performed by Viroscan 3D (Lyon University, Faculté de Médecine et de Pharmacie, 69,008 Lyon). Paired-end $2 \times 150$ bp sequencing was conducted on an Illumina NextSeq500 instrument, with a High Output 150-cycle kit. CLC Genomics Workbench (Version 9.5.2, Qiagen Bioinformatics) was used to assemble 9,529,152 (mean length $149 \mathrm{bp}$ ) and 9,834,144 reads (mean length $149 \mathrm{bp}$ ) for strains S1 and B16, respectively. Final sequencing coverages were $143 \mathrm{x}$ and 150x with 51 and 53 scaffolds for strains S1 and B16, respectively.

\section{Annotation and comparison of the Dickeya genomes}

The functional annotation of predicted genes was achieved using Rapid Annotation Subsystem Technology (RAST) server (http://rast.nmpdr.org/rast.cgi, Aziz et al. [17]) with the Glimmer 3 gene caller [18]. Genes of interest (Tables 3, S1, S2 and S3) were manually annotated and assigned to functional classes with the help of the Psi-Blast server. 


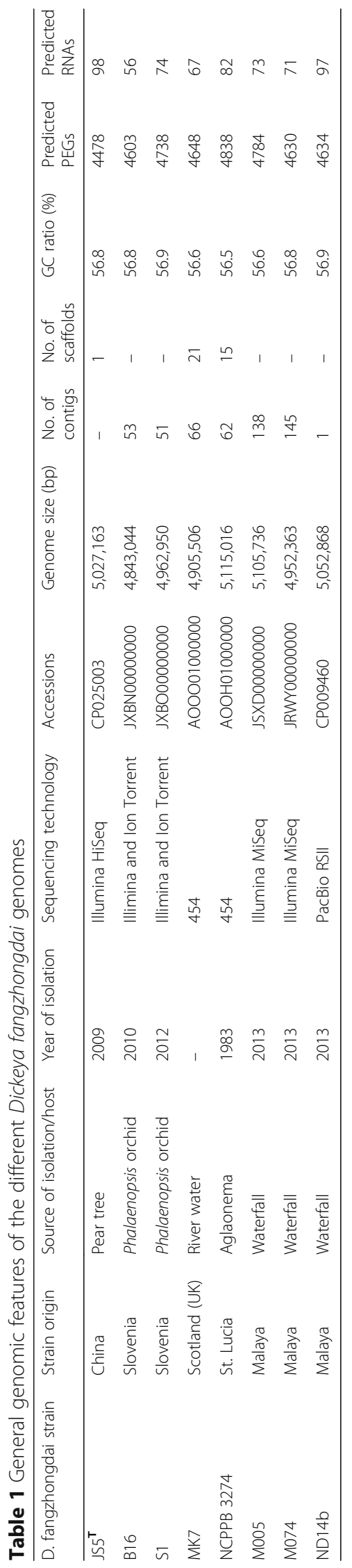


Average nucleotide identity (ANI) was computed using the JSpecies package version 1.2.1 with the MUMer algorithm (http://jspecies.ribohost.com/jspeciesws/). In silico DNA-DNA Hybridization (DDH) was calculated according to [19], using a dedicated pipeline (http:// ggdc.dsmz.de/).

The pan-genome (the entire set of gene families found in genomes of a species), core genome (set of gene families shared by all strains of a species), and species-specific genes (genes unique to one species) were determined using a homology constraint of $80 \%$ amino acid identity and $80 \%$ alignment coverage. Two R scripts were used to calculate rarefaction (core genome) and accumulation (pan genome) curves, as described by Meric et al. [20].

As we were working with draft genomes, some split or truncated genes were noted in the final genome assembly. Additional analysis of the annotated draft genomes was conducted to eliminate sequencing errors for the genes of interest (virulence genes, secondary metabolite pathways, specific genes). Presence and position of those genes was manually inspected to detect split genes, truncated genes at the end of contigs and missing genes.

Visualization and comparison of genomes was conducted on the CGView server (Circular Genome Viewer, https://server.gview.ca/) with the BlastAtlas tool [21]. Two complete genomes (ND14b and JS5) were used as reference genomes. Further, genome-to-genome comparison was conducted using bi-directional protein-protein BLAST sequence comparison of translated open reading frames (ORFs) with a $10^{-5}$ e-value threshold. Genes were considered as strain-specific if the identity of the encoded protein was lower than $80 \%$ of the full-length amino acids sequence of the longest protein.

\section{Phylogenetic analysis}

In silico multilocus sequence analysis (MLSA) was performed on 1162 concatenated amino acid orthologous sequences. Clustering of orthologous sequences into homologous families was conducted using the SiLix software package [22], using an $80 \%$ identity threshold on full-length proteins. Orthologous sequences were aligned with the Muscle software [23], then concatenated, and alignments were curated using Gblocks [24]. Phylogeny was performed using the PhyML algorithm [25] with the following settings: substitution model LG, 100 bootstraps, model-given amino acid equilibrium frequencies, invariable sites optimized, tree searching NNI, starting tree BioNJ. Pectobacterium atrosepticum was used as an outgroup. The iTOL tool was used to visualize the phylogenetic trees [26].

Identification of mobile and extrachromosomal elements The prophage identification tool PHAge Search Tool Enhanced Release (PHASTER) was used to determine the region containing prophage-like elements in bacterial genomes (http://phaster.ca/) [27]). Prediction of clustered regularly interspaced short palindromic repeats (CRISPRs) was done using CRISPRfinder (http://crispr.i2bc.paris-saclay.fr/Server/) [28]. Insertion sequences and genomic islands were identified using the ISfinder (https://isfinder.biotoul.fr/general_information.php) [29] and IslandViewer 4 (http://www.pathogenomics.sfu.ca/ islandviewer/) [30], respectively.

\section{Presence of virulence-associated genes}

The presence of Dickeya virulence-associated genes previously reported by Reverchon and Nasser [4] was assessed using BLASTp analysis. The D. fangzhongdai core genome was compared to $D$. dadantii 3937 genome annotation from the ASAP database [31] to determine the presence of known virulence determinants and regulators. Furthermore, genomic regions containing secondary metabolite biosynthesis gene cluster were identified using AntiSMASH software (version 4.1.0, https:// doi.org/10.1093/nar/gkv437) [32].

\section{Functional classification of the specific genes}

D. fangzhongdai species-specific gene families i. e. families absent in or sharing below $80 \%$ homology with the gene families present in other Dickeya species, were extracted from the SiLix output.

The COG categorization of species-specific genes was conducted by submitting predicted CDS to the EggNOG 4.5 database [33] and subsequent extraction of COG categories. Furthermore, signatures for protein families, domains and repeats was determined using Psi-Blast [34]. The predictions were performed on the basis of $D$. fangzhongdai ND14b or JS5 gene sequences. The manual functional classification of the species-specific genes was conducted on the basis of RAST annotation, COG categorisation, Psi-blast and structure signatures information.

\section{Antibiotic resistance}

Susceptibility to antibiotic streptomycin was tested for D. fangzhongdai strains S1, B16, MK7, JS5, and NCPPB 3274. The bacteria were grown on Luria Bertani (LB) medium with $1.5 \%$ agar. Bacterial suspensions were prepared in $10 \mathrm{mMPB}$ buffer $(1.07 \mathrm{~g}$ $\mathrm{Na}_{2} \mathrm{HPO}_{4}, 0.4 \mathrm{~g} \mathrm{NaH} \mathrm{PO}_{4} \cdot 2 \mathrm{H}_{2} \mathrm{O}$ per liter of water, $\mathrm{pH}$ 7.2) from overnight cultures to the final concentration of $10^{6} \mathrm{cfu} / \mathrm{mL}$. Antibiotic resistance was tested by spot plating $15 \mu \mathrm{L}$ of bacterial suspensions to $\mathrm{LB}$ medium plates containing streptomycin (ranging from 5 to $100 \mu \mathrm{g} / \mathrm{ml}$ ). Inoculated plates were incubated overnight at $28^{\circ} \mathrm{C}$. 


\section{Results}

\section{Statistics and features of the genomes}

This study included the eight genomes of $D$. fangzhongdai that were available in GenBank databases. The Nd14b genome has been completely sequenced using PacBio technology [35]. We built improved versions of B16 and S1 strains genomes using Illumina technology, which resulted in the availability of seven draft genomes that comprise approximately 1-150 contigs, precluding synteny studies of the $D$. fangzhongdai species (Table 1 ). ANI and DDH analyses confirmed the assignation of the eight strains into the same species and their closeness to the D. solani/D. dadantii/D. dianthicola clade (Fig.1). This is consistent with the MLSA phylogenetic analysis (Fig. 2).

As already shown by DNA-DNA hybridizations [14], the NCPPB 3274 strain is at the limit of being part of the $D$. fangzhongdai species. Indeed, if the ANI values exceeded 96\% threshold, the DDH values range from $68.6-70.5 \%$.

\section{D. fangzhongdai core and accessory genomes}

To determine the species core and pan genomes, we conducted a comparative genomic analysis of $D$. fangzhongdai genomes annotated by the RAST platform, using the SiLix gene family clustering tool. Proteins were classified as homologous to another in a given family if the amino acid identity were above $80 \%$ on the full-length amino acid sequence. The core genome of $D$. fangzhongdai, defined as gene families shared by all strains of the species, comprises 3520 gene families and represents roughly three-quarters of all predicted encoding proteins in each strain. This core genome is highly conserved, since $86 \%$ of the core genome genes share at least $95 \%$ identity. The species pan-genome, defined as the entire set of gene families found in the genomes of the species, includes 7249 gene families that represent twice the number of the core genome genes. This continuously increases with the addition of each genome, thereby indicating that the D. fangzhongdai pan-genome is still far from closeness (Fig. 3).

\section{Most known Dickeya virulence determinants are present in $D$. fangzhongdai}

Several virulence factors have been characterized in the D. dadantii 3937 model strain. Soft rot symptoms are caused by the production and secretion of plant cell wall degrading enzymes, pectinases and cellulases secreted by the Out T2SS (type 2 secretion system) and proteases secreted by the Prt T1SS. Furthermore, an efficient colonization of the host plant requires the production of several additional bacterial factors that are either involved in the adhesion and penetration into plant tissues or in the adaptation to the different stresses encountered by the bacteria inside plants. These factors include cell envelope components (LPS and EPS), motility, efficient iron uptake systems, as well as defences against acidic and oxidative stresses or against antibacterial compounds produced by the plant [4]. All D. fangzhongdai strains possess the entire battery of plant cell wall degrading enzymes present in D. dadantii 3937 and their cognate secretion systems; they also harbour the second

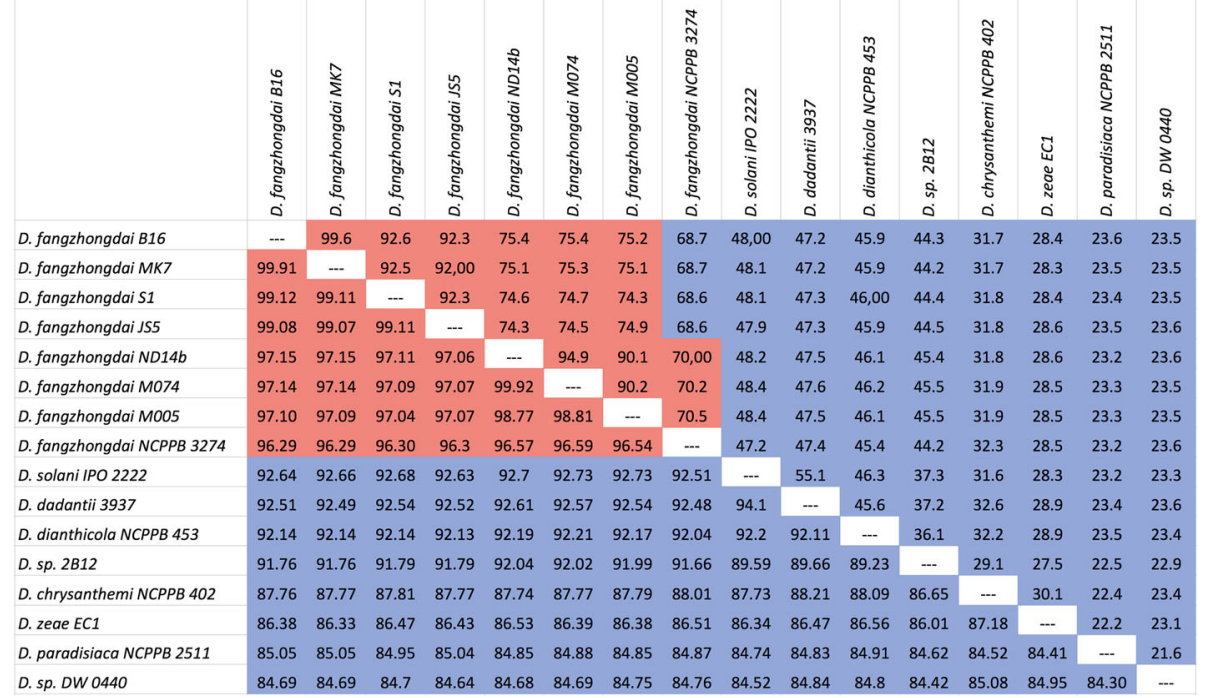

Fig. 1 Definition of the Dickeya fangzhongdai species in silico DNA-DNA hybridization (DDH, upper triangle) and Average Nucleotide Identity (ANI, lower triangle) values of Dickeya fangzhongdai strains and representative strains of the other described Dickeya species. Strains belonging to the same species are highlighted in red. The specific threshold value is $96 \%$ for ANI and $70 \%$ for DDH 


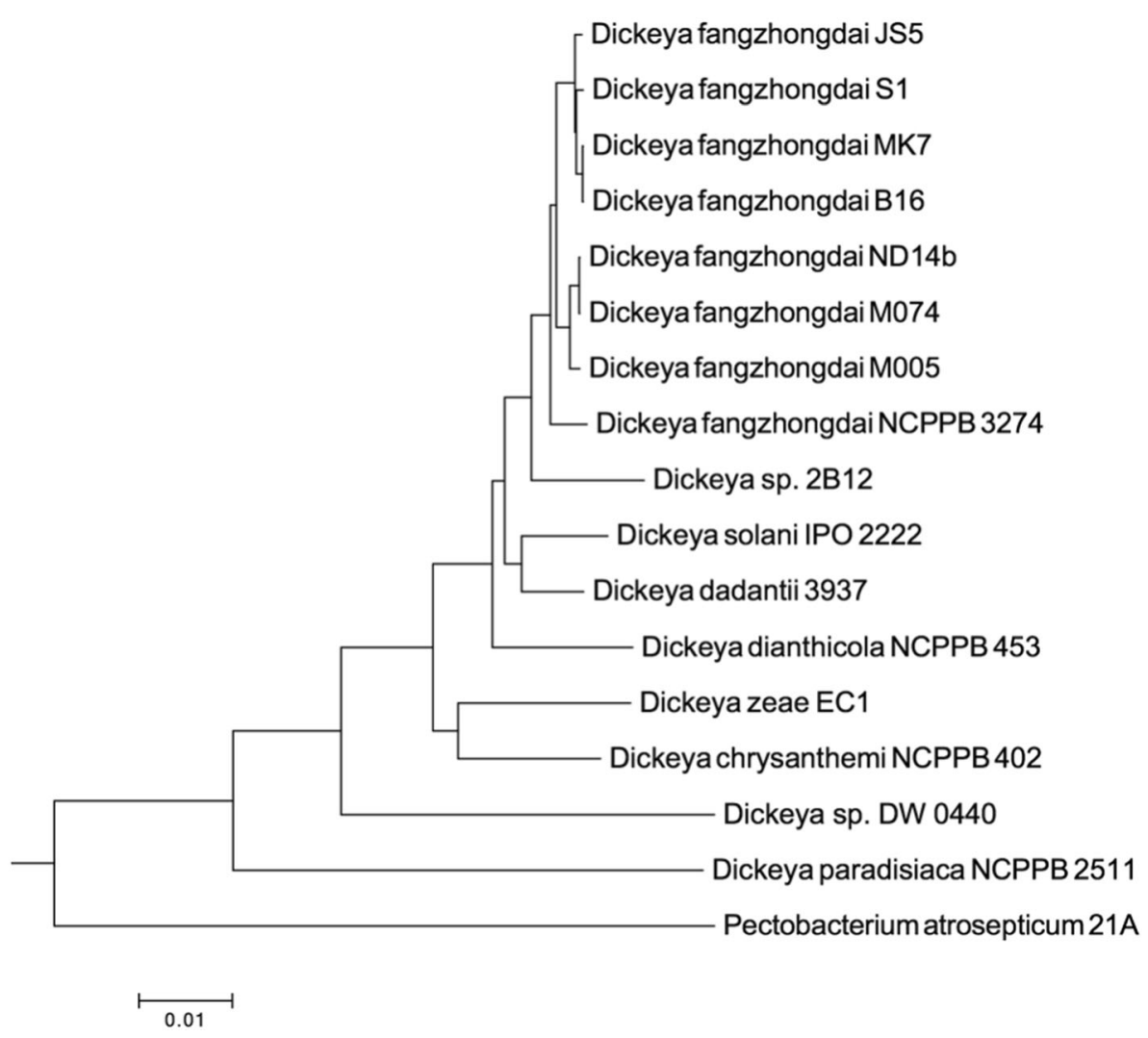

Fig. 2 Phylogenetic tree of Dickeya fangzhongdai strains and representative strains of other described Dickeya species. The tree was constructed from concatenated sequences of 1162 homologous amino acid sequences (71,912 variable sites). One hunderd bootstrap replicates were conducted to assess the statistical support of each node. Bootstrap support values are $100 \%$ for all nodes but one. Pectobacterium atrosepticum 21 A was used as an outgroup

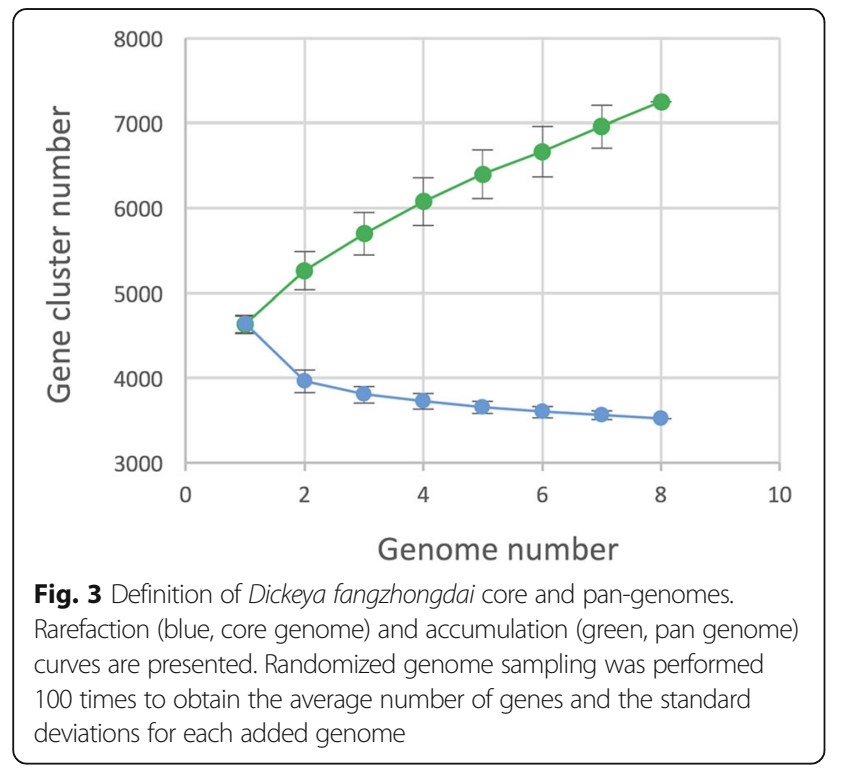

Stt T2SS system that is present in $D$. dadantii but is not widely spread among the different Dickeya species. The $D$. fangzhongdai core genome also includes the vast majority of the other bacterial factors shown to be involved in interactions with plants in $D$. dadantii [4], with two exceptions: the gene encoding the acid shock periplasmic Asr protein that plays a role in survival under acid conditions is missing in all D. fangzhongdai strains as do the $i a a M H$ genes involved in auxin production. However, the presence of these genes in Dickeya genomes varied within the genus.

\section{Diversity in other protein secretion systems}

Apart from the T1SS and T2SS systems, all $D$. fangzhongdai strains possess a T3SS and share the same repertoire of T3SS effectors. More diversity was observed for the T4SS, T5SS and T6SS.

Two types of T4SS might be present in bacteria, either associated with plasmid conjugation and DNA uptake/ release or to protein secretion [36]. D. dadantii 3937 encodes both types of T4SS, a VirD2/VirD4/Trb locus present in an integrative conjugative transposon element 
(ICE) and another one regrouping only a $\operatorname{virB}$ operon [37]. Conserved VirD2/VirD4/Trb systems are present in D. fangzhongdai ND14b, M074 and M005. The M005 locus is highly syntenic with $D$. dadantii 3937 , while in the two other strains it is present in a different common genetic context. In addition, like 3937, ND14b, M074 and M005 possess a virB operon. A virB operon is also present in D. fangzhongdai S1, and in two copies in $D$. fangzhongdai NCPPB 3274. No virD4 or virD2 genes are present in these two strains, however noticeably, in certain bacteria like Bordetella pertussis, a T4SS was shown to be functional for protein secretion even in the absence of a VirD4 homolog [38]. Finally, D. fangzhongdai strains MK7 and B16 did not contain any genes connected to T4SS, and JS5 possesses only a virD4-related gene that is located near a pil-tra gene cluster that is predicted to be involved in plasmid conjugative transfer. Therefore, among the eight analysed D. fangzhongdai strains, only five harbour T4SS and three of them additionally possess a module involved in conjugation.

Type V (T5SS) and type VI (T6SS) secretion systems are both involved in contact-dependent inter- and intra-species competition systems. T5SS are two-partner secretion systems called Hec, Tsp or Cdi (contact-dependent inhibition) that consist of an outer membrane $\mathrm{TspB} / \mathrm{HecB}$ protein allowing the secretion of a large TspA/HecA multidomain protein. TspA/HecA protein comprises an $\mathrm{N}$-terminal transport domain, a large hemagglutinin-like region that is proposed to form a fibre-like structure and typically a C-terminal toxin domain beginning with the VENN motif [39].

D. dadantii 3937 harbours two T5SS systems that have been shown to act in contact-dependent growth inhibition $[39,40]$. In Nd14 and JS5, the only D. fangzhongdai complete genomes available, one hecB and two hecA genes, were identified in regions showing high synteny with the related $D$. dadantii 3937 regions. In ND14b, both HecA proteins are 59 and 63\% identical (76 and 77\% similar) to their counterparts in 3937; the C-terminal toxin portion is different in these four proteins (Fig. 4). The JS5 HecA proteins are even more similar to the 3937 proteins (66 and 73\% identical, 84-87\% similar). All other D. fangzhongdai strains possess a hecB homolog. The detection of HecA encoding genes is ambiguous since the reads assembly is often interrupted in these very long (up to 4000 amino acids) modular proteins that contain a high number of repeats. Most of the genes are truncated at the end of contigs leading to truncated predicted proteins. Nevertheless, indications of the presence of hecA1 and hecA2 genes were found in all $D$. fangzhongdai but the MK7 genome (Additional file 2: Table S1).

T6SS systems are contractile nanomachines that function in a manner analogous to an inverted phage tail and tube to deliver effectors in target cells. They comprise the secretion machinery encoded by the imp/vas operon, the hemolysin-coregulated protein ( $\mathrm{Hcp}$ ) and valine-glycine repeat protein $\mathrm{G}$ (VgrG) secreted proteins that form a membrane puncturing device, and effectors such as so called Rearrangement HotSpot (Rhs) proteins. The Rhs proteins are large composite proteins consisting of a large $\mathrm{N}$-domain that contain YD-peptide repeats and a highly variable $\mathrm{C}$-domain that harbours toxic activity. To block the toxic activity of the Rhs protein, a small immunity protein RhsI is produced, whose encoding gene is very often located just adjacent to the corresponding rhs gene [40]. The rhs genes are very often located close to $h c p$-vgrG genes. D. dadantii 3937 possess three copies of these hcp-vgrG-rhs clusters and one of them is

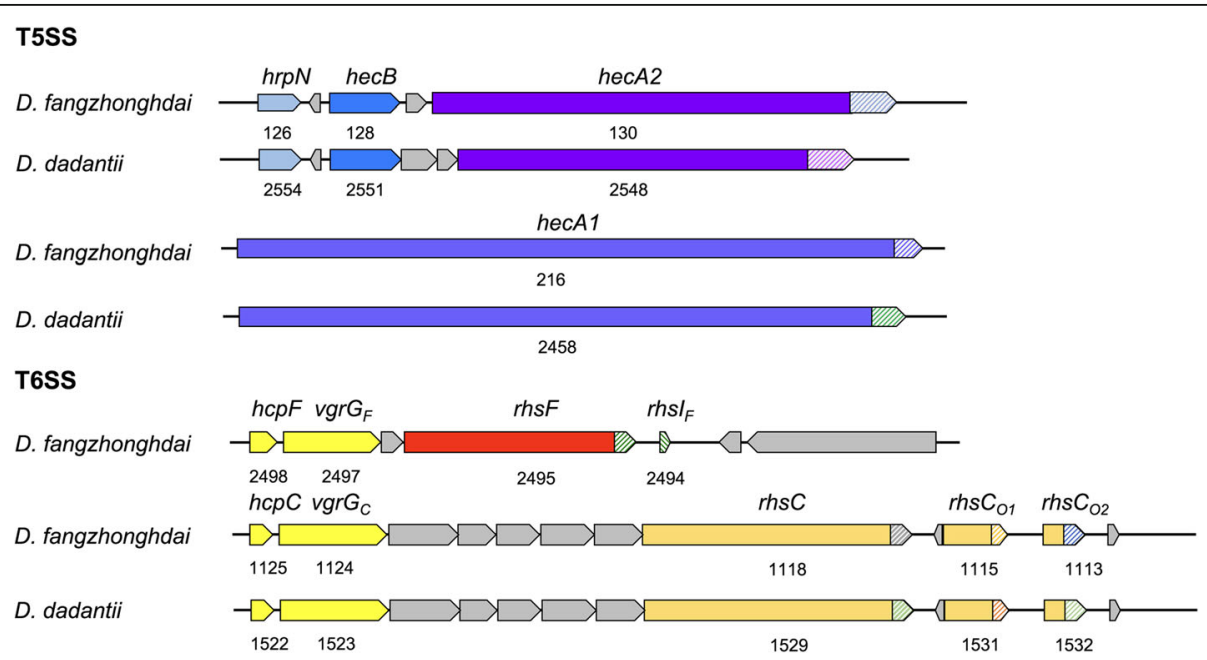

Fig. 4 Genes or genetic clusters of T5SS and T6SS-related toxin/antitoxin systems in the model Dickeya fangzhongdai strain ND14b. The different clusters were compared to the corresponding clusters present in the 3937 Dickeya dadantii model strain. The rhsF cluster is found only in the Dickeya fangzhongdai species even if it is highly similar to the hcp-vgrG-rhs $B_{3937}$ cluster (see text for details) 
Table 2 Secondary metabolite gene clusters identified with AntiSMASH in Dickeya fangzhongdai genomes

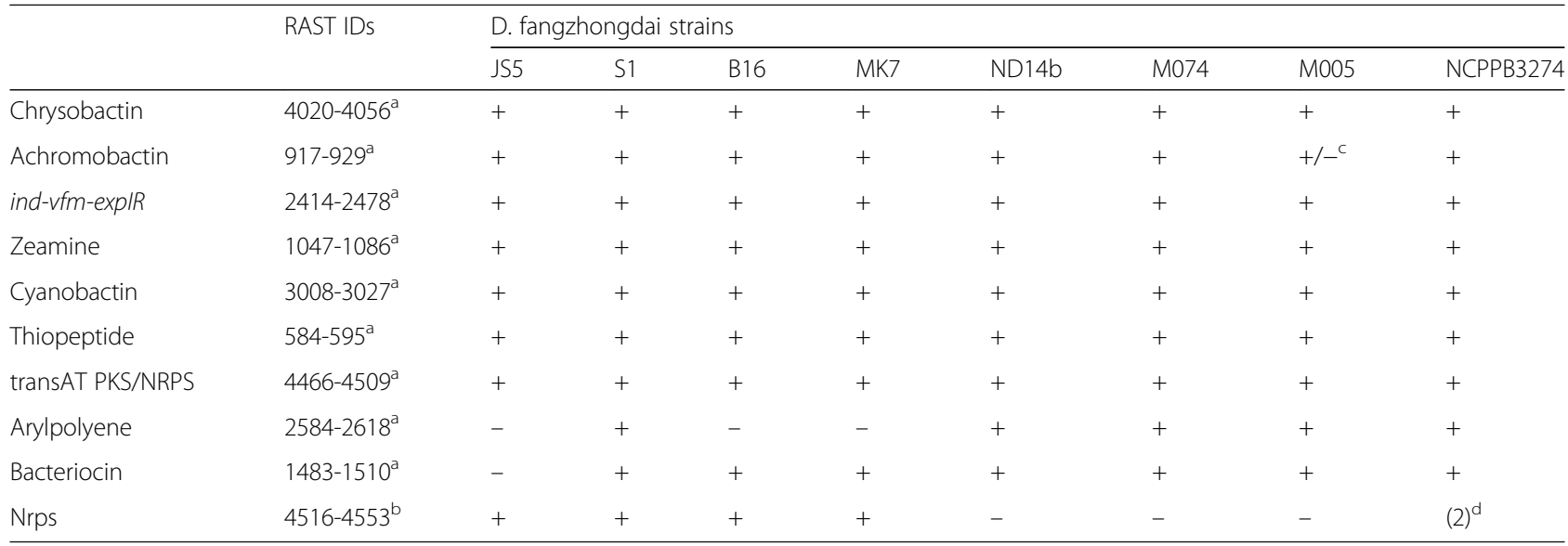

aenes based on the ND14b Rast ID

${ }^{\mathrm{b}}$ genes based on the JS5 Rast ID

'genome assembly problems for this region in $\mathrm{M} 005$

${ }^{d}$ NCPPB3274 harbours two additional NRPS clusters, different from the cluster found in JS5, S1, B16 and MK7

+ , presence of the genetic cluster; $+/-$, partial gene cluster; - , absence of the genetic cluster

accompanied by a number of additional orphan Rhs-CT/ RhsI pairs. All D. fangzhongdai strains possess a well-conserved entire imp/vas operon. The Hcp-vgrG$r h s C_{3937}$ cluster is largely conserved in all strains, even if genome assembly problems clouded the identification of some of these genes (Additional file 2: Table S1). In ND14b, the N-end of the RhsC and the orphan-related proteins are highly similar to their 3937 counterparts; however, the CT regions are different (Fig. 4). On the contrary, the $h c p-v g r G-r h s B_{3937}$ cluster was identified only in $D$. fangzhongdai NCPPB3274 and S1. We did not detect any homo$\log$ to the $h c p-v g r G-r h s A_{3937}$ cluster in the same genomic location in $D$. fangzhongdai; however, interestingly, all $D$. fangzhongdai strains possess another hcp-vgrG-rhs cluster named $h c p$-vgrG-rhsF in which the RhsF protein is highly similar to the $h c p-v g r G-r h s B_{3937}$ (88\% identity/95\% similarity even in the $\mathrm{CT}$ region) (Fig. 4).

\section{Secondary metabolites and corresponding pathways}

Analysis with the antiSMASH server [32] identified seven secondary metabolite biogenesis clusters in the $D$. fangzhongdai core genome, as summarized in Table 2 . Three of these clusters encode the biosynthesis of well-known secondary metabolites produced by all Dickeya analysed so far. The chrysobactin and achromobactin clusters govern the biosynthesis of the two siderophores responsible for the efficient uptake of iron and are involved in bacterial survival and virulence [4]. The ind-vfm-expI cluster groups genes that are involved in the synthesis of the antioxidant indigoidine molecule as well as in the quorum sensing regulation mediated by an AHL and the new VFM signal [2, 41]; the production of indigoidine by $D$. fangzhongdai was experimentally confirmed in previous studies [11]. All D. fangzhongdai strains also possess the gene cluster involved in the biosynthesis of the zeamine toxin. Two other secondary metabolite-related clusters were predicted to be involved in the biosynthesis of either cyanobactin-related or thiopeptide-related molecules. Cyanobactins are small cyclic peptides with variable functions, the most common activity is cytotoxic [42]. Several thiopeptides present an antibiotic activity [43]. However, we could not connect these clusters to any known molecule. The last cluster common to all $D$. fangzhongdai strains is encoding a non-ribosomal peptide synthase (NRPS) and polyketide synthases (PKS) complex. These NRPS/PKS complexes are involved in the synthesis of polymers of peptidyl/carbonyl chains. In this cluster, Nd14b possesses an additional and rather long NRPS encoding gene that indicates the production of slightly different polymers than in other $D$. fangzhongdai strains.

In addition to the seven clusters described above, shared by all $D$. fangzhongdai, further five clusters detected by AntiSMASH were found only in some $D$. fangzhongdai strains-two of them were predicted to comprise genes involved in the synthesis of a bacteriocin or the biosynthesis of arylpropylene. The bacteriocin synthesis cluster is present in all $D$. fangzhongdai strains, except JS5. The arylpoliene biosynthesis cluster was identified in all D. fangzhongdai strains, excluding MK7, JS5 and B16 isolates. The third cluster encodes an NRPS cluster that is shared by the JS5, B16, S1 and MK7 strains. Since no homologous genetic cluster could be identified in any other bacterial genome in databases, no information on the produced secondary metabolite is available. NCPPB3274 harbours two additional NRPS clusters. One of them encodes a trans-acyltransferase polyketide synthase cluster that presents the same 
genetic organization as the oocydin A clusters found in Serratia plymuthica and Serratia marcescens strains.

\section{Virulence regulatory pathways}

D. fangzhongdai species showed homology to $D$. dadantii 3937 virulence genes, and only minor differences were noted in their content. This is also the case for the regulatory proteins regulating the virulence programme. Indeed, all the regulators identified in $D$. dadantii 3937 as controlling virulence factors [4] were present in the core genome of the $D$. fangzhongdai species, including global regulators, nucleotide-associated proteins and post-transcriptional regulation molecules.

\section{Species-specific genes might be important for species virulence}

To extract unique features of the $D$. fangzhongdai species, a genome comparison was conducted by comparing the $D$. fangzhongdai core genome with the genomes of 31 strains belonging to the seven other Dickeya species (marked on the MLSA tree, in Fig. S1). Species-specific genes are defined as present in all D. fangzhongdai strains and absent in all other Dickeya species (using a specific threshold of $80 \%$ identity on the full-length protein sequence). Only 38 gene families specific to $D$. fangzhongdai species were detected, thereby confirming the high genetic conservation of the Dickeya genus. Approximately one-half of these genes encode hypothetical proteins (19 proteins with 9 smaller than 60 amino acids). These species-specific genes were not clustered together in a region of the genome; rather, they were evenly scattered throughout the genome. Table 3 presents the characteristics of the $25 \mathrm{D}$. fangzhongdai-specific genes for which a function is predicted or genes that have protein motives and/or genes that were found in other bacteria genera. A significant proportion of the species-specific genes with predicted function are genes involved in regulation (four genes) and different types of metabolism (six genes). Another species-specific gene encodes a short orphan NRPS protein (512 AA) that did not correspond to any known secondary metabolite biosynthesis pathway.

Interestingly, D. fangzhongdai contains an additional and unique pectate lyase gene. The encoding protein differs significantly from the pectate lyase found in other bacterial plant pathogens or in other Dickeya spp., except in D. chrysantemi Ech 1591 (74\% protein sequence identity). Based on protein structure prediction, this protein belongs to family 10 of polysaccharide lyase (PL10). A signal peptide was identified to be 27 AA long, thereby indicating extracellular or periplasmic localisation of the protein. Since PL10 from D. chrysanthemi Ech 1591 has not been characterised yet, no other properties of the protein are available.

\section{Genomic diversity in the $D$. fangzhongdai strains}

\section{Fangzhongdai clades}

As visualized in the MLSA phylogenetic tree (Fig.2), ANI/ DDH values clearly indicate additional clustering within the $D$. fangzhongdai species. Indeed, the strains can be divided into three branches-a JS5-like and a ND14b-like cluster and the more divergent NCPPB3274 strain (Fig. 1). For both clusters, the genome conservation between the members of the same cluster is extremely high. Indeed, strains within the JS5-like and ND14b-like cluster shared on average of 85 and $88 \%$ of the gene families, respectively; moreover, approximately $95 \%$ of these genes were at least 95\% identical. Each clade shares a pool of 90-160 genes that are shared by the clade members but are absent from the other D. fangzhongdai strains. Interestingly, most of these genes were not present in other Dickeya genomes (Additional file 3: Tables S2 and S3). However, approximately one-half of these clade-shared genes mainly comprised short hypothetical proteins that were below $60 \mathrm{AA}$ long (49 genes in the JS5-like and 61 genes in the ND14b-like clade). The remaining genes corresponded to (i) metabolism, including secondary metabolite biogenesis, (ii) transport, (iii) regulation or (iv) other functions (Additional file 3: Tables S2 and S3; Additional file 1: Figure $\mathrm{S} 1$ ). In contrast to species-specific genes, several of these genes shared by clade members are clustered in genomic regions (GR), and several of these clustered genes have a predicted function (Table 4). Three genomic regions were identified in the JS5-like clade. The GR1 gene content indicates an involvement in carbon metabolism. GR2 groups three very large proteins of unknown functions and a gene involved in transport. GR3 contains a NRPS biosynthesis cluster operon, as already described above.

Five genomic regions were identified in the ND14b-like clade. Three of them (GR4, 6 and 7) comprised mainly genes related to metabolism and transport. The GR7 sequence is not conserved in other bacteria. In contrast, homologs of GR4 and GR6 were found in Leclercia sp. LSNIH3 and D. dadantii 3937, respectively. GR5 comprises the second VirD2/VirD4/Trb T4SS system described above. Biologically the most interesting genomic region is GR8. It contains five genes that code for a sigma-fimbriae structure. No homologous region was found in other bacteria. However, based on the genetic structure of the region, it is related to the $c s u$-like operon of Pseudomonas aeruginosa. CsuA/BABCDE pili normally comprise six proteins; however, only five related proteins were found in the ND14b-like clade. Therefore, protein sequences of the GR6 cluster were compared to the well-defined CsuA/BABCDE pili proteins from Acidovorax baumannii 19606 to identify the putative functions of the proteins in GR6. The GR6 proteins share 58-73\% similarity with their A. baumannii strain 19606 counterparts, namely CsuE (gene ID 3899), CsuD (gene ID 3900), 


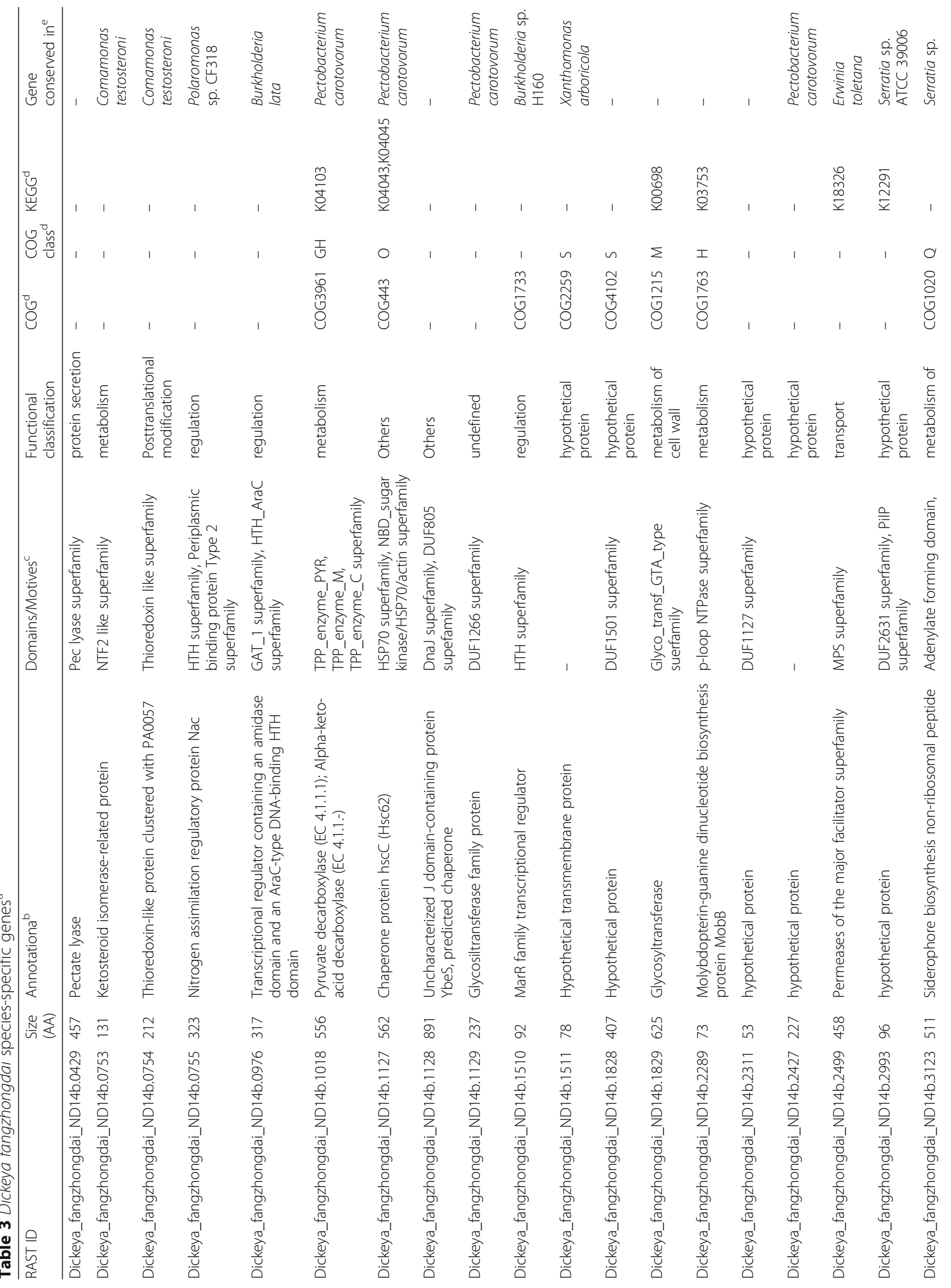




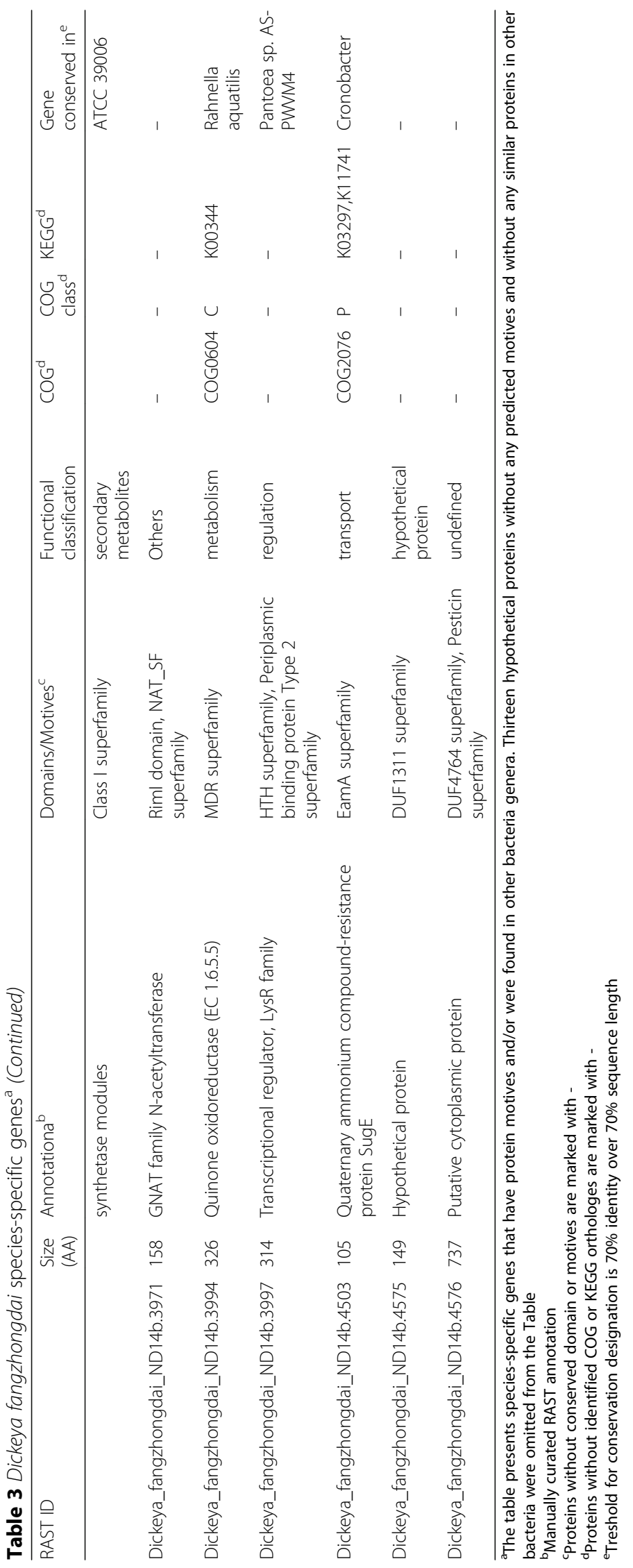


Table 4 Genomic regions of the JS5 and ND14b genomic clades

\begin{tabular}{|c|c|c|c|c|c|c|}
\hline Clade & Genomic region & Size of GR (nt) & Genes RAST ID & $\begin{array}{l}\text { Presence of horizontal } \\
\text { transfer signature }\end{array}$ & Predicted function & Region conserved in ${ }^{c}$ \\
\hline \multirow[t]{3}{*}{ JS5-like clade ${ }^{a}$} & GR1 & 7033 & $3204-3211^{a}$ & No & metabolism (putative) & D. dianthicola RNS04.9 \\
\hline & GR2 & 11,303 & $3941-3945^{a}$ & No & Transport (putative) & - \\
\hline & GR3 & 12,717 & $4354-4357^{\mathrm{a}}$ & No & NRPS & - \\
\hline \multirow[t]{5}{*}{ ND14b-like clade ${ }^{b}$} & GR4 & 4729 & $317-323^{b}$ & No & metabolism (putative) & Leclercia sp. LSNIH3 \\
\hline & GR5 & 11,197 & $1736-1746^{b}$ & Yes & ICE (partial) & D. dadantii 3937 \\
\hline & GR6 & 11,493 & $2589-2598^{b}$ & No & metabolism (putative) & D. dadantii 3937 \\
\hline & GR7 & 9241 & $2906-2913^{b}$ & No & metabolism (putative) & - \\
\hline & GR8 & 4732 & $3899-3903^{b}$ & No & type I pilus & - \\
\hline
\end{tabular}

agenes based on the JS5 Rast ID

${ }^{b}$ genes based on the ND14b Rast ID

cthe best hit in BLASTn analysis

Genomic regions found in each of the JS5 and Nd14b genomic clades that are absent from the other Dickeya fangzhongdai genomes are presented. Conserved

regions share at least $70 \%$ identity over $70 \%$ sequence length

CsuC (gene ID 3901) and CsuA/B (ID 3903), predicted as tip adhesin, chaperone, usher protein and major pili subunit, respectively. However, one of the minor tip subunits CsuA or CsuB is missing in the ND14b-clade GR6. All the ND14b-clade corresponding proteins were predicted as non-cytoplasmic (TMHMM), although only the protein sequences encoded by the ID3902 and ID3899 genes contained a signal peptide, as predicted by SignalP4.1 (http:// www.cbs.dtu.dk/services/SignalP/) [44] and Phobius (http://phobius.sbc.su.se/) [45] programs. Furthermore, the protein sequences encoded by the ID3902 and ID3901 genes possessed a predicted transmembrane region like the A. baumannii CsuA and CsuC proteins, respectively. A transmembrane region was also identified in the protein sequence 3900, although the corresponding CusD protein contains a signal peptide rather than a transmembrane sequence in the same region (TMHMM, SignalIP4.1). Based on our results, members of the Nd14b-like clade have genetic dispositions for type I like pili expression; however, functional pili have to be experimentally confirmed. The JS5-like clade members did not contain a $c s u A / B A B C D E$ operon; however, few very short fragments of the pili proteins (below 100 AA) were conserved. For Acinetobacter baumannii 19606, it was shown that the csu locus is involved in bacteria attachment and biofilm formation on abiotic surfaces [46].

\section{D. fangzhongdai diversity resides mainly in genes of extrachromosomal origin}

To further analyse the diversity between $D$. fangzhongdai stains, we performed a Blast-Atlas analysis based on the complete genomes of one representative of each clade, ND14b and JS5 (Fig. 5). This revealed that, in both clades, the conserved genes are evenly spread over the entire genome and that M074 is very close to ND14b. Only a few genomic regions of the Nd14b/M074 and JS5 genomes are not present in any of the other $D$. fangzhongdai isolates. The two large Nd14b/M074 1.86-1.88 Mbp and 4.56-4.62 Mbp regions encode prophages. Four regions are present only in JS5. The $0.45 \mathrm{Mb}$ and $4.1 \mathrm{Mb}$ regions consist of genes encoding mobile elements and hypothetical proteins. The large $0.51-0.52 \mathrm{Mb}$ region comprises 77 genes related to transposition, conjugative transfer systems and replication typical of Integrative and Conjugative Elements (ICE). The last region $(0.9 \mathrm{Mb})$ groups genes that are either involved in metabolism or encoding mobile elements. The high accumulation of genes encoding extrachromosomal elements in our BLAST-Atlas analyses prompted us to identify such genes in each $D$. fangzhongdai genome (Table 5). The number of sequences related to mobile elements varies among the $D$. fangzhongdai isolates. The genomes were relatively poor with insertion sequences (IS), harbouring none (S1 genome) to up to five full IS elements per genome, as identified by the ISfinder tool. The elements were members of four different IS families, namely IS200/IS605, IS110, IS30, IS4 and IS3. Different combinations of elements were present but all genomes with IS elements contained an IS200/IS605 family (IS200 group). Most of the analysed D. fangzhongdai strains did not contain complete prophage sequences within their genomes, but only possess short prophage related fragments. Nevertheless, an intact prophage was found within the ND14b and M074 genomes and two different prophages in the NCPPB 3274 genome as detected by the Phaster server. One of the $D$. fangzhongdai NCPPB 3274 prophages corresponds to the one found in $D$. dadantii 3937 , as $83 \%$ of the prophage region exhibited above $90 \%$ nucleotide identity. Sequences homologous to the second NCPPB 3274 prophage are present in D. zeae EC1 ( $89 \%$ of the prophage region exhibited above $90 \%$ nucleotide identity). Both prophages sequences corresponded to Myoviridae bacteriophages, namely to Haemophilus virus HP1 and HP2 and to Enterobacteria phage P88, respectively. CRISPR elements are very 


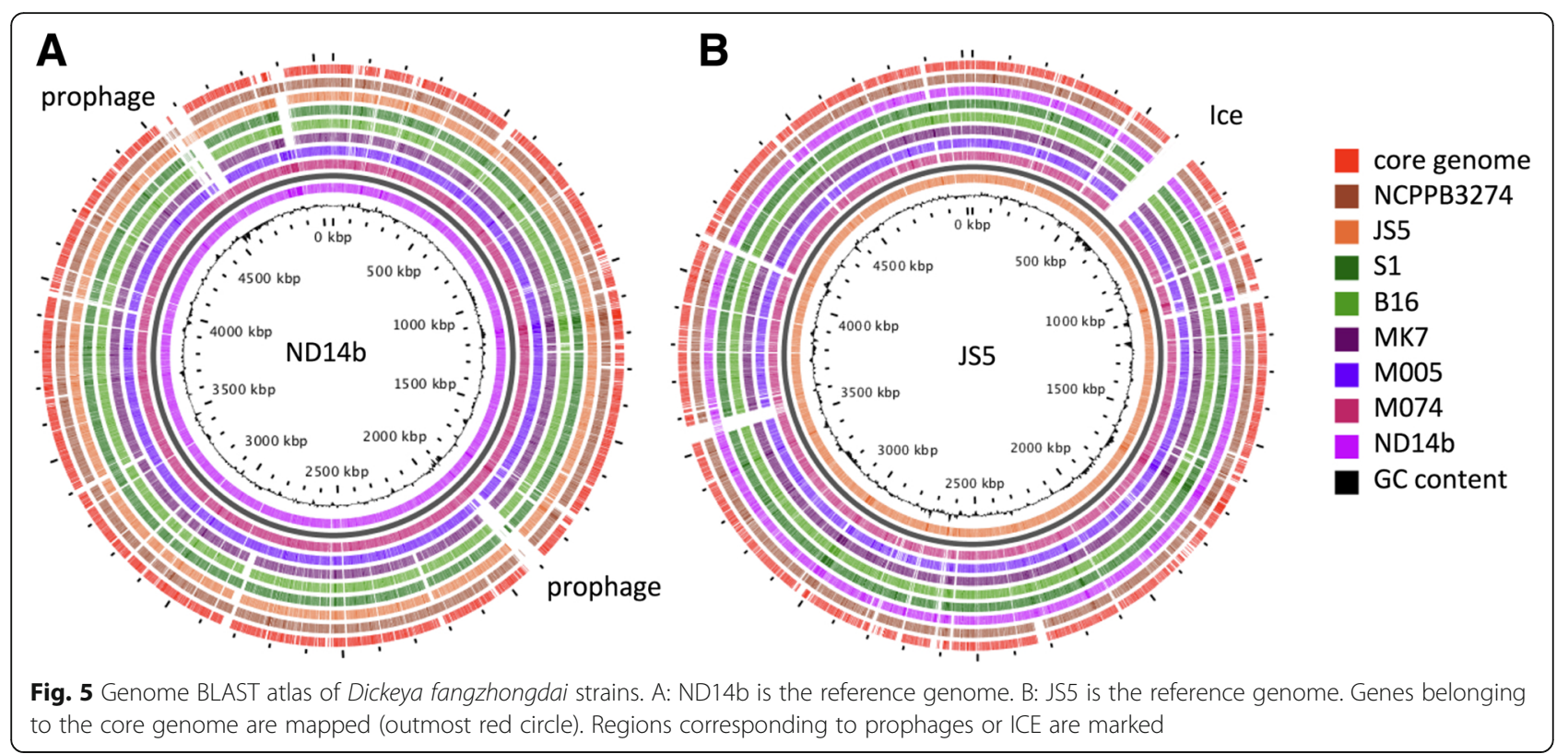

important features of bacterial genomes as they provide acquired immunity against viruses and plasmids [47]. All D. fangzhongdai genomes comprised multiple CRISPR sequences. The number of CRISPRs varies from four to seven complete CRISPRs among the isolates (CRISPRfinder tool), a variation also encountered in other Dickeya isolates and species. Furthermore, the S1 strain harbours an entire contig (contig 24) that presents all characteristics of a plasmid even if we did not succeed in closing it (see below). NCPPB 3274 also harbours a $92 \mathrm{~Kb}$ long contig that contains ten genes involved in conjugative transfer (related to IncF-traBCNUHI and a relaxase) and encoding a ParA plasmid partitioning protein. The other genes mainly encode hypothetical proteins and the contig sequence was not homolog to any bacterial or plasmid sequences. In the M005 strain, the IslandViewer server identified a large genomic island (ID_M005.4077 to 4134) that groups integrase genes as well as plasmid-like replication and recombination functions, as well as a conjugative machinery typical of
ICEs related to the Pseudomonas fluorescens Pf-5 PFGI-1 genomic island [48]. Up to two other genetic clusters related to ICE elements, mainly to ICE elements found in other Dickeya spp. (ICEDda3937-1, ICEDdaEch586-1 and ICEDzeEch1591-1), were found in most D. fangzhongdai genomes, excluding the MK7 and B16 strains.

\section{The strain S1 plasmid}

The $23 \mathrm{~Kb}$-long S1 contig 24 regroups several plasmidrelated proteins involved in replication (gene ID2790), plasmid stabilization (genes ID2773, 2787) and genes related to conjugative transfer of large self-transmissible broad-host range RP4-type IncP plasmids. The IncP transfer system comprises two regions, Tra1 and Tra2, which code for the DNA transfer and replication system (Dtr encoded by traC to $N, 12$ proteins) and the mating pair formation (Mpf encoded by trbA to $P, 15$ proteins) apparatus involved in bringing the donor and the recipient cells into intimate contact during conjugation [49].

Table 5 Presence of different types of mobile elements and CRISPR in Dickeya fangzhongdai genomes

\begin{tabular}{|c|c|c|c|c|c|}
\hline Dickeya fangzhongdai strain & $\begin{array}{l}\text { No. of confirmed } \\
\text { CRISPR elements }\end{array}$ & No. of intact prophages & $\begin{array}{l}\text { No. of incomplete } \\
\text { or questionable prophages }\end{array}$ & No. of full IS elements & IS families \\
\hline JS5T & 5 & - & 1 & 3 & IS200/IS605, IS110, IS3 \\
\hline B16 & 7 & - & 8 & 3 & IS200/IS605, IS30, IS3 \\
\hline S1 & 3 & - & 6 & 0 & - \\
\hline MK7 & 7 & - & 5 & 2 & IS200/IS605, IS30 \\
\hline NCPPB 3274 & 6 & 2 & 1 & 3 & IS200/IS605, IS110, IS4 \\
\hline M005 & 4 & - & 7 & 4 & IS200/IS605, IS30 \\
\hline M074 & 5 & 1 & 4 & 4 & IS200/IS605, IS110, IS3 \\
\hline ND14b & 4 & 1 & 2 & 5 & IS200/IS605, IS110, IS3 \\
\hline
\end{tabular}


Thus, the S1 plasmid transfer machinery is incomplete since it contains only six genes that encode TraKJI proteins involved in binding to the OriT origin of transfer, TrbJK proteins involved in the entry exclusion mechanism avoiding transfer of other plasmids of the IncP group and TrbL that is a TraG/VirB6 homolog involved in the biogenesis of the T4SS transfer pilus. Nevertheless, the pS1 plasmid is highly similar to the p3-T1 plasmid described in Acidovorax sp. T1, even if the p3-T1 plasmid is substantially larger $(56.4 \mathrm{kbp})$ than pS1 (23 kbp). A comparison between these two plasmids revealed that all genes related to plasmid maintenance, replication and conjugation are conserved (Fig. 6), but that both plasmids diverge in genes involved in metabolic pathways. Indeed, the $\mathrm{p} 3-\mathrm{T} 1$ plasmid carries an operon involved in mercuric resistance and several genes that encode enzymes and genes that are related to mobile elements that do not have counterparts in pS1. At the same location as the mercury resistance genes, the $\mathrm{pS} 1$ sequence contains two streptomycin kinases genes, namely $\operatorname{stra} A$ and $\operatorname{str} B$, which are responsible for resistance to the streptomycin antibiotic. In bacterial isolates from plants, strA-strB genes are often encoded on the Tn3-type transposon Tn5393 that is generally borne on conjugative plasmids [50]. No full transposable elements were detected in the S1 putative plasmid sequence. Nevertheless, a partial Tn3-type transposon that includes the genes encoding the TniABQ transposase-related proteins is present. The functionality of the streptomycin-resistant genes present in $\mathrm{pS} 1$ was tested. The S1 strain was able to grow on LB plates supplemented with streptomycin concentration up to $100 \mu \mathrm{g} / \mathrm{mL}$. No other tested Dickeya strain exhibited streptomycin resistance even at antibiotic concentrations as low as $5 \mu \mathrm{g} / \mathrm{ml}$.

\section{Discussion}

D. fangzhongdai, the last described Dickeya spp., is genomically close to the $D$. dadantii, $D$. dianthicola and $D$. solani clade [14]. Members of this clade are mainly associated to soft rot disease of herbaceous plants. However, $D$. fangzhongdai appears to have a wider range of habitats since it was reported to be not solely a soft rot pathogen of herbaceous plants, but is also connected to bleeding canker symptoms of pear trees and seems to be common in aquatic environments [9-11]. To analyse the possible links between genomic characteristics and observed diversity, we explored the signature genetic traits of the species and intra-species variability.

The comparative genomic analysis of eight $D$. fangzhongdai genomes revealed a large intra-species diversity. Indeed, the Average Nucleotide Identity values ranged from 99.9 to $96 \%$ and seven of the eight genomes can be divided into two separate clades. While the three strains of the ND14b-like cluster were all isolated from the same environment (water) in the same country, the other cluster grouped strains isolated from very different environments (water and monocot or dicot diseased plants) and from a wide geographical area (Table 1). However, a recent study on a former Erwinia chrysanthemi collection isolated in Japan showed that $D$. fangzhongdai strains isolated from diseased monocot plants are distributed between both clades $[10,14]$ indicating that genomic relatedness and habitat are not correlated.

The $D$. fangzhongdai core genome comprises over 3500 genes, a number comparable to other core genomes of the Dickeya species. Indeed, as determined in our Silix analysis, the size of the core genome is similar to that of the phylogenetically close $D$. dianthicola and $D$. dadantii species, for which the core genome includes 3711 (5 genomes analysed) and 3386 (4 genomes analysed) gene families, respectively. The $D$. solani core genome is larger (4009 gene families) due to the high genetic homogeneity of the $D$. solani strains sequenced so far $[37,51,52]$. This highlights the high genetic conservation of the Dickeya genus. Indeed, the core genome is much larger than those of other enterobacterales species, like Escherichia coli, that includes only around 1500 orthologous genes [53]. This high conservation is also exemplified by the fact that the vast majority of virulence genes and genes involved in the complex regulatory network that

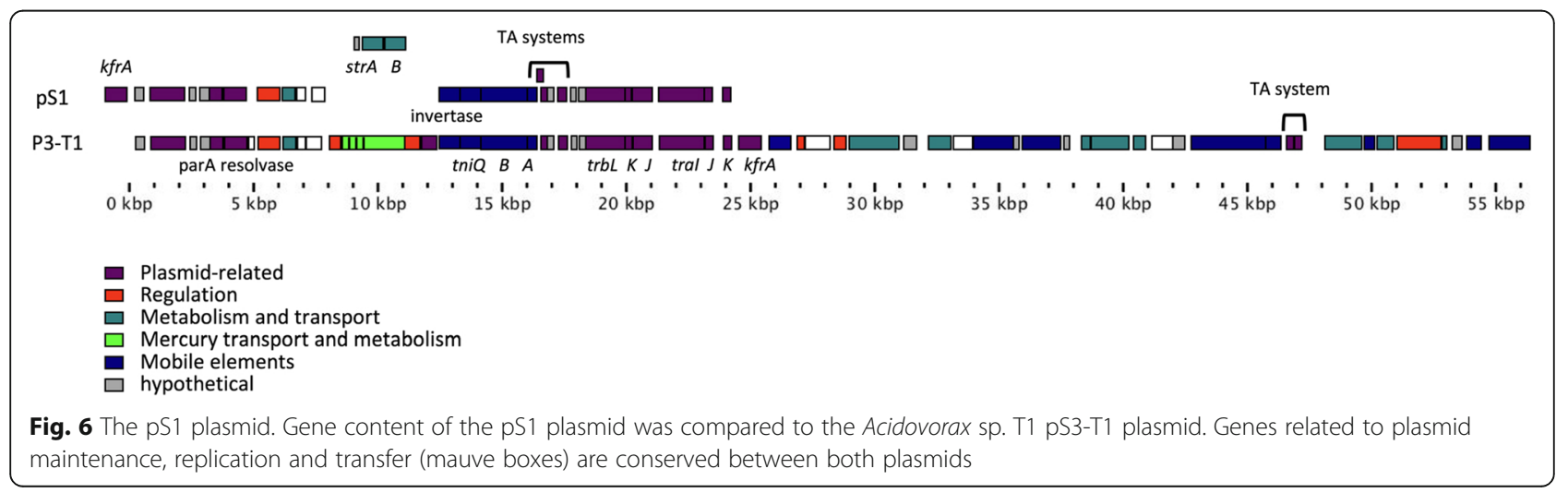


controls virulence that are well characterized in other Dickeya species, are also conserved in D. fangzhongdai.

Only a few dozen genes are specific to $D$. fangzhong$d a i$, thereby confirming the high genetic conservation of the Dickeya genus. This number of species-specific gene families is rather low since our SiLix analysis revealed that other Dickeya species contain from 100 to 200 species-specific gene families Interestingly, all analysed D. fangzhongdai strains shared a few specific regulatory genes and a gene that encodes an additional pectate lyase. This pectinase belongs to the PL10 family and no members of this family were reported in the Dickeya genus, except in D. chrysanthemi Ech 1591. Pectate lyases of the family PL10 are found exclusively in a few bacteria from the plant environment, soil or human gut. $D$. fangzhongdai is the only enterobacteral species that contains a pectate lyase from PL10 family in its core genome [54]. Since some D. fangzhongdai strains showed higher aggressiveness and maceration potential compared to other Dickeya species [11], it would be interesting to analyse whether the presence of this additional pectate lyase and observed high maceration levels are connected.

D. fangzhongdai harbours several clusters encoding the biosynthesis of secondary metabolites that are involved in defence against stresses or the production of toxic compounds that may be important during plant-bacteria interactions. So, all analysed D. fangzhongdai strains harbour genes involved in the biosynthesis of zeamine, thiopeptide, cyanodactin or a NRPS/PKS cluster. Interestingly, zeamine was initially identified in the rice pathogen $D$. zeae $\mathrm{EC} 1$ as a phytotoxic virulence factor and is also active as an antibacterial agent [55]. In contrast to the zeamine produced by Serratia, the compounds produced by $D$. fangzhongdai strains have however very low nematode-killing activities [56]. Nevertheless, zeamine production does not appear to be restricted to Dickeya strains virulent on monocots, since D. solani also possesses a similar cluster [37]. Others of these genes clusters are shared by only some of the $D$. fangzhongdai strains. So, several D. fangzhongdai strains encode genes involved in the production of aryl polyene compounds that are structurally similar to the wellknown carotenoids, and like these compounds, some of them were shown to protect the bacterium from reactive oxygen species [57]. Some strains also encode genes involved in oocydin A and a bacteriocin. Oocydin A is an anticancer haterumalide with strong antimicrobial activity against agriculturally important plant pathogenic fungi and oomycetes [58-60]. Similar cluster sequences (above $80 \%$ identity) are also present in other Dickeya sp., $D$. solani, various $D$. dianthicola, $D$. zeae $\mathrm{EC} 1$, and D. paradisiaca Ech703. Bacteriocins are small antibioticlike compounds with bactericidal activity that is usually restricted to closely related species or strains, thereby increasing competition during infection [61]. They are common in Gram-negative bacteria [62]. D. fangzhongdai strains also encode additional NRPS/PKS complexes for which the synthesized products are not known. Other genes known to involved in bacteria-bacteria interactions and to provide a selective advantage to $D$. fangzhongdai in various environments are T5SS and T6SS effectors. It is worth to note that the C-terminal toxic parts of these effectors are very diverse between the different $D$. fangzhongdai strains (Fig. 4). All these secondary metabolites and effectors are known or predicted to harbour several biological activities such as adaptation to unfavourable environments or interspecific competition [63] and may contribute to the persistence of $D$. fangzhongdai in diverse environments.

In addition to these secondary metabolite biosynthesis pathways, intra-species $D$. fangzhongdai diversity resides mainly in the presence of genes of extrachromosomal origin since $D$. fangzhongdai strains carry diverse elements such as prophages, ICEs and plasmids. Plasmids are very rare in Dickeya spp. since, out of the 60 genome assemblies available in NCBI databases, only two plasmids have been identified in Dickeya spp.-pS1 described here and a plasmid present in D. solani strain 9019 that is also present in Burkholderia [51] and carries genes involved in antibiotic resistance. All these elements may contribute to the rapid evolution of these bacterial pathogens via horizontal gene transfer.

One of the purposes of this study was to analyse if genomic comparisons may identify traits that would differentiate strains isolated from different environments (plants versus water, monocots versus dicots) or explain the phenotypic differences reported between various $D$. fangzhongdai strains [14]. Our analyses however did not provide us with any clues to enlighten experimentally observed phenotypic differences or habitat specificity. Indeed, no genetic traits that would explain previously reported intra- and inter-species phenotype diversity [14] were identified. In the same way, we were unable to link observed differences in carbon metabolism with differences in genomic gene contents. Examination of genes that are present in JS5 and absent in all other $D$. fangzhongdai isolates (Additional file 4: Table S4) identified only 227 genes, mostly coding for hypothetical proteins (55\%) and genes of extrachromosomal origin (23\%). No gene clusters or metabolic pathways, that would be linked to host adaptation, could be identified. Neither were the species-specific genes abundant in regulatory or regulation-connected genes $(2 \%)$.

Similarly, search for genes that are present only in $D$. fangzhongdai strains isolated from water sources did not reveal any specific genetic traits that could be associated to adaptation to the aquatic environment. Water-isolates 
shared only two short hypothetical genes that were not present in other $D$. fangzhongdai members.

One possible clue for explaining these differences in habitat or phenotypic capacities might be subtle variations in the regulatory networks involved in metabolic abilities, since various strain-specific genes encode regulatory proteins and furthermore, the observed high conservation of the genes involved in the virulence regulatory network does not imply a similar regulation of virulence genes. Indeed, variations in the degree of control exerted by master regulators have been observed in different $D$. solani strains with significant differences in aggressiveness [64]. Alternatively, our results may reflect the ubiquity of $D$. fangzhongdai to adapt to various environments and to infect very diverse hosts. Indeed, some $D$. fangzhongdai strains isolated from monocots were shown to be highly aggressive on dicots like potato tubers [11]. The next steps to unravel these questions would be an in-depth determination of the virulence of different $D$. fangzhongdai isolates on a large plant panel and the analysis of expression profiles in different growth conditions or during infection of diverse plant hosts.

\section{Conclusions}

D. fangzhongdai isolates were found in different habitats like monocot and dicot plants and waterways. Comparison of the genome information of eight members of this new species isolated from these diverse environments revealed a high proportion of species core genome genes (three quarters of total genomes). These include the majority of virulence genes and virulence-related global regulators characterized in other Dickeya species. Importantly, it also allows the identification of a few dozen genes specific to D. fangzhongdai that provide a basis for the development of DNA-based effective detection and diagnosis.

The intra-species diversity of the D. fangzhongdai species resides mainly in secondary metabolites biosynthetic pathways, T5SS and T6SS-related toxins or the repertoire of genes of extrachromosomal origin and also in gene clusters related to metabolism and transport. However, no genetic traits that would differentiate strains isolated from different environments and contribute to habitat specificity could be identified. This might reflect the ubiquity of D. fangzhongdai to adapt to various environments including fresh water and infect very diverse hosts. D. fangzhongdai may thus spread via waterways and constitute a potential threat to several economically important crops.

\section{Additional files}

Additional file 1: Figure S1. Dickeya strains used in comparative genomics. (DOCX $1173 \mathrm{~kb}$ )

Additional file 2: Table S1. T5SS and T6SS effectors present in the different Dickeya fangzhongdai genomes. (DOCX 25 kb)
Additional file 3: Tables S2 and S3. Genes found in each of the JS5 and Nd14b genomic clades that are absent from the other Dickeya fangzhongdai genomes. (ZIP $37 \mathrm{~kb}$ )

Additional file 4: Table S4. Genes present in the JS5 strain that are absent in all other Dickeya fangzhongdai strains. (XLSX $17 \mathrm{~kb}$ )

\section{Abbreviations}

ANI: Average Nucleotide Identity; bp: Base pairs; CDI: Contact-dependent immunity; DDH: In silico DNA-DNA Hybridization; ICE: Integrative and conjugative element; NRPS: Non-ribosomal peptide synthase; PKS: Polyketide synthase; rhs: Recombination hot spot; TxSS: Type $x$ secretion system

\section{Acknowledgements}

The authors thank lan Toth and Sonia Humphries of the James Hutton Institute for the Dickeya fangzhongdai MK7 strain, and Xiang Li, Canadian Food Inspection Agency, for useful communication.

\section{Funding}

This study was funded by the European Regional Development Fund; the Republic of Slovenia's Ministry of Higher Education, Science and Technology; Slovenian Research Agency (contracts P4-0165 and 1000-15-0105), Public Scholarship, Development, Disability and Maintenance Fund of the Republic Slovenia (Ad Futura scholarship); COST Action FA1208 and the Euphresco Phytosanitary ERA-NET project on Dickeya and Pectobacterium spp. This study was also supported by the French Agence Nationale de la Recherche (ANR15-CE21-0003).

\section{Availability of data and materials}

The genomic sequences generated and/or analysed during the current study are available in GenBank database at the NCBI server (https:// www.ncbi.n/m.nih.gov/genbank/), with the accession numbers provided in Table 1 and Additional file 1: Figure S1. Gene predictions and automatic functional annotations are available in the RAST server (http://rast.nmpdr.org/ rast.cgi). Manual annotation corrections are presented in Tables 3 and Additional file 3: S2 and S3 Additional file 4: S4.

\section{Authors' contributions}

FVG and TD conceived and designed the experiments, SA and JP contributed to conception and design of the experiments, SA, JP and FVG performed analysis and interpretation of data, SA performed the identification of secondary metabolic pathways and extrachromosomal elements. All authors participated in comparative genomic data production and in the writing of the manuscript. All authors read and approved the final manuscript

Ethics approval and consent to participate

Not applicable.

\section{Consent for publication}

Not applicable.

Competing interests

The authors declare that they have no competing interests.

\section{Publisher's Note}

Springer Nature remains neutral with regard to jurisdictional claims in published maps and institutional affiliations.

\section{Author details}

${ }^{1}$ National Institute of Biology, Vecna pot 111, SI-1000 Ljubljana, Slovenia. ${ }^{2}$ Jozef Stefan International Postgraduate School, Jamova 39, SI-1000 Ljubljana, Slovenia. ${ }^{3}$ Institute of Ecology and Environmental Sciences of Paris, Sorbonne Universités, UPMC Univ Paris 06, Diderot Univ Paris 07, UPEC Univ Paris 12, CNRS, INRA, IRD, 4 Place Jussieu, 75005 Paris, France. 
Received: 1 August 2018 Accepted: 29 November 2018 Published online: 11 January 2019

\section{References}

1. Adeolu M, Alnajar S, Naushad S, Gupta RS. Genome-based phylogeny and taxonomy of the 'Enterobacteriales': proposal for enterobacterales Ord. Nov. divided into the families Enterobacteriaceae, Erwiniaceae fam. Nov., Pectobacteriaceae fam. Nov., Yersiniaceae fam. Nov., Hafniaceae fam. Nov., Morgane. Int J Syst Evol Microbiol. 2016;66:5575-99.

2. Charkowski A, Blanco C, Condemine G, Expert D, Franza T, Hayes C, et al. The role of secretion systems and small molecules in soft-rot Enterobacteriaceae pathogenicity. Annu Rev Phytopathol. 2012;50:425-49.

3. Joko T, Subandi A, Kusumandari N, Wibowo A, Priyatmojo A. Activities of plant cell wall-degrading enzymes by bacterial soft rot of orchid. Arch Phytopathol Plant Prot. 2014:47:1239-50.

4. Reverchon S, Nasser W. Dickeya ecology, environment sensing and regulation of virulence programme. Environ Microbiol Rep. 2013;5:622-36.

5. Ma B, Hibbing ME, Kim H-S, Reedy RM, Yedidia I, Breuer J, et al. Host range and molecular phylogenies of the soft rot enterobacterial genera Pectobacterium and Dickeya. Phytopathology. 2007:97:1150-63.

6. Samson R, Legendre JB, Christen R, Fischer-Le Saux M, Achouak W, Gardan L. Transfer of Pectobacterium chrysanthemi (Burkholder et al. 1953) Brenner et al. 1973 and Brenneria paradisiaca to the genus Dickeya gen. Nov. as Dickeya chrysanthemi comb. nov. and Dickeya paradisiaca comb. nov. and delineation of four novel species. Int J Syst Evol Microbiol. 2005;55:1415-27.

7. van der Wolf JM, Nijhuis EH, Kowalewska MJ, Saddler GS, Parkinson N, Elphinstone JG, et al. Dickeya solani sp. nov., a pectinolytic plant-pathogenic bacterium isolated from potato (Solanum tuberosum). Int I Syst Evol Microbiol. 2014;64:768-74.

8. Parkinson N, DeVos P, Pirhonen M, Elphinstone J. Dickeya aquatica sp. nov., isolated from waterways. Int J Syst Evol Microbiol. 2014;64:2264-6.

9. Tian $Y$, Zhao $Y$, Yuan $X, Y i$ J, Fan J, Xu Z, et al. Dickeya fangzhongdai sp. nov., a plant pathogenic bacterium isolated from pear trees (Pyrus pyrifolia). Int J Syst Evol Microbiol. 2016;66:2831-5.

10. Suharjo R, Sawada H, Takikawa Y. Phylogenetic study of Japanese Dickeya spp. and development of new rapid identification methods using PCR-RFLP. J Gen Plant Pathol. 2014:80:237-54.

11. Alič NT, Tušek-Žnidarič M, Peterka M, Ravnikar M, Dreo T. Putative new species of the genus Dickeya as major soft rot pathogens in Phalaenopsis orchid production. Plant Pathol. 2017;66:1357-68.

12. Onwueme I. Taro cultivation in Asia and the Pacific. Rap Publication. 1999;16:1-9.

13. Galeano Mendoza CH, Baquero Cubillos EF, Molina Varón JA. Cerón lasso M del S. agronomic evaluation of bunching onion in the Colombian cundiboyacense high plateau. Int. J Agron. 2018;2018:1-8.

14. Alič Š, Van Gijsegem F, Pédron J, Ravnikar M, Dreo T. Diversity within the novel Dickeya fangzhongdai sp., isolated from infected orchids, water and pears. Plant Pathol. 2018;67:1612-20.

15. Parkinson N, Stead D, Bew J, Heeney J, Tsror L, Elphinstone J. Dickeya species relatedness and clade structure determined by comparison of recA sequences. Int J Syst Evol Microbiol. 2009;59:2388-93.

16. Alič $\breve{S}$, Naglič T, Llop P, Toplak N, Koren S, Ravnikar M, et al. Draft genome gequences of Dickeya sp. isolates B16 (NIB Z 2098) and S1 (NIB Z 2099) causing coft rot of Phalaenopsis orchids. Genome Announc. 2015;3:e00973-15.

17. Aziz RK, Bartels D, Best AA, DeJongh M, Disz T, Edwards RA, et al. The RAST server: rapid annotations using subsystems technology. BMC Genomics. 2008;9:75.

18. Delcher AL, Bratke KA, Powers EC, Salzberg SL. Identifying bacterial genes and endosymbiont DNA with glimmer. Bioinformatics. 2007 Mar 15;23:673-9.

19. Meier-Kolthoff JP, Auch AF, Klenk H-P, Göker M. Genome sequence-based species delimitation with confidence intervals and improved distance functions. BMC Bioinformatics. 2013;14:60

20. Méric G, Yahara K, Mageiros L, Pascoe B, Maiden MCJ, Jolley KA, et al. A reference pan-genome approach to comparative bacterial genomics: identification of novel epidemiological markers in pathogenic Campylobacter. Bereswill S, editor PLoS One. 2014;9:e92798.

21. Stothard P, Grant JR, Van Domselaar G. Visualizing and comparing circular genomes using the CGView family of tools. Brief Bioinform. 2017. https:// doi.org/10.1093/bib/bbx081.

22. Miele V, Penel $\mathrm{S}$, Duret L. Ultra-fast sequence clustering from similarity networks with SiLiX. BMC Bioinformatics. 2011;12:116.
23. Edgar RC. MUSCLE: multiple sequence alignment with high accuracy and high throughput. Nucleic Acids Res. 2004;32:1792-7.

24. Castresana J. Selection of conserved blocks from multiple alignments for their use in phylogenetic analysis. Mol Biol Evol. 2000;17:540-52.

25. Guindon S, Dufayard J-F, Lefort V, Anisimova M, Hordijk W, Gascuel O. New algorithms and methods to estimate maximum-likelihood phylogenies: assessing the performance of PhyML 3.0. Syst Biol. 2010;59: $307-21$.

26. Letunic I, Bork P. Interactive tree of life (iTOL) v3: an online tool for the display and annotation of phylogenetic and other trees. Nucleic Acids Res. 2016:44:W242-5.

27. Arndt D, Grant JR, Marcu A, Sajed T, Pon A, Liang Y, et al. PHASTER: a better, faster version of the PHAST phage search tool. Nucleic Acids Res. 2016;44: W16-21.

28. Grissa I, Vergnaud G, Pourcel C. CRISPRFinder: a web tool to identify clustered regularly interspace short palindromic repeats. Nucleic Acids Res. 2007;35:52-7.

29. Siguier P, Perochon J, Lestrade L, Mahillon J, Chandler M. ISfinder: the reference Centre for bacterial insertion sequences. Nucleic Acids Res. 2006; 34:D32-6.

30. Bertelli C, Laird MR, Williams KP, Lau BY, Hoad G, Winsor GL, et al. IslandViewer 4: expanded prediction of genomic islands for larger-scale datasets. Nucleic Acids Res. 2017;45:W30-5.

31. Glasner JD, Liss P, Plunkett G III, Darling A, Prasad T, Rusch M, et al. ASAP, a systematic annotation package for community analysis of genomes. Nucleic Acids Res. 2003;31:147-51.

32. Blin K, Wolf T, Chevrette MG, Lu X, Schwalen CJ, Kautsar SA, et al. AntiSMASH 4.0-improvements in chemistry prediction and gene cluster boundary identification. Nucleic Acids Res. 2017:45:W36-41.

33. Huerta-Cepas J, Szklarczyk D, Forslund K, Cook H, Heller D, Walter MC, et al. EggNOG 4.5: a hierarchical orthology framework with improved functional annotations for eukaryotic, prokaryotic and viral sequences. Nucleic Acids Res. 2016;44:D286-93.

34. Altschul S, Madden TL, Schäffer AA, Zhang J, Zhang Z, Miller W, et al. Gapped BLAST and PSI-BLAST: a new generation of protein database search programs. Nucleic Acids Res. 1997;25:3389-402.

35. Zhao Y, Tian Y, Li X. Hu B. complete genome sequence of a Dickeya fangzhongdai type strain causing bleeding canker of pear tree trunks. Genome Announc. 2018;6:e00177-18.

36. Wallden K, Rivera-Calzada A, Waksman G. Type IV secretion systems: versatility and diversity in function. Cell Microbiol. 2010;12:1203-12.

37. Pédron J, Mondy S, Raoul des Essarts Y, Van Gijsegem F, Faure D. Genomic and metabolic comparison with Dickeya dadantii 3937 reveals the emerging Dickeya solani potato pathogen to display distinctive metabolic activities and T5SS/T6SS-related toxin repertoire. BMC Genomics. 2014;15:1-13.

38. Alvarez-Martinez CE, Christie PJ. Biological diversity of prokaryotic type IV secretion systems. Microbiol Mol Biol Rev. 2009;73:775-808.

39. Aoki SK, Diner EJ, De Roodenbeke CTK, Burgess BR, Poole SJ, Braaten BA et al. A widespread family of polymorphic contact-dependent toxin delivery systems in bacteria. Nature. 2010;468:439-42.

40. Koskiniemi S, Lamoureux JG, Nikolakakis KC. t'Kint de Roodenbeke C, Kaplan $M D$, low $D A$, et al. Rhs proteins from diverse bacteria mediate intercellular competition. Proc Natl Acad Sci U S A. 2013;110:7032-7.

41. Nasser W, Dorel C, Wawrzyniak J, Van Gijsegem F, Groleau M-C, Déziel E, et al. Vfm a new quorum sensing system controls the virulence of Dickeya dadantii. Environ Microbiol. 2013;15:865-80.

42. Sivonen K, Leikoski N, Fewer DP, Jokela J. Cyanobactins-ribosomal cyclic peptides produced by cyanobacteria. Appl Microbiol Biotechnol. 2010;86: 1213-25.

43. Bagley MC, Dale JW, Merritt EA, Xiong X. Thiopeptide antibiotics. Chem Rev. 2005;105:685-714.

44. Petersen TN, Brunak S, von Heijne G, Nielsen H. SignalP 4.0: discriminating signal peptides from transmembrane regions. Nat Methods. 2011:8:785-6.

45. Kall L, Krogh A, Sonnhammer ELL. Advantages of combined transmembrane topology and signal peptide prediction--the Phobius web server. Nucleic Acids Res. 2007;35:W429-32.

46. Tomaras AP, Dorsey CW, Edelmann RE, Actis LA. Attachment to and biofilm formation on abiotic surfaces by Acinetobacter baumannii: involvement of a novel chaperone-usher pili assembly system. Microbiology. 2003;149:3473-84. 
47. Horvath P, Barrangou R. CRISPR/Cas, the immune system of bacteria and archaea. Source Sci New Ser. 2010;327:167-70.

48. Mavrodi DV, Loper JE, Paulsen IT, Thomashow LS. Mobile genetic elements in the genome of the beneficial rhizobacterium Pseudomonas fluorescens Pf5. BMC Microbiol. 2009;9:8.

49. Grohmann E, Muth G, Espinosa M. Conjugative plasmid transfer in grampositive bacteria. Microbiol Mol Biol Rev. 2003;67:277-301.

50. Sundin GW, Bender CL. Dissemination of the strA-strB streptomycinresistance genes among commensal and pathogenic bacteria from humans, animals, and plants. Mol Ecol. 1996;5:133-43.

51. Khayi S, Blin P, Pédron J, Chong TM, Chan KG, Moumni M, et al. Population genomics reveals additive and replacing horizontal gene transfers in the emerging pathogen Dickeya solani. BMC Genomics. 2015;16:1-13.

52. Pritchard L, Humphris S, Saddler GS, Parkinson NM, Bertrand V, Elphinstone $J G$, et al. Detection of phytopathogens of the genus Dickeya using a PCR primer prediction pipeline for draft bacterial genome sequences. Plant Pathol. 2013;62:587-96.

53. Lukjancenko O, Wassenaar TM, Ussery DW. Comparison of 61 sequenced Escherichia coli genomes. Microb Ecol. 2010;60:708-20.

54. Hugouvieux-Cotte-Pattat N, Condemine G, Shevchik VE. Bacterial pectate lyases, structural and functional diversity. Environ Microbiol Rep. 2014;6:427-40.

55. Zhou J, Zhang H, Wu J, Liu Q, Xi P, Lee J, et al. A novel multidomain polyketide synthase is essential for zeamine production and the virulence of Dickeya zeae. Mol Plant-Microbe Interact. 2011;24:1156-64.

56. Hellberg JEEU, Matilla MA, Salmond GPC. The broad-spectrum antibiotic, zeamine, kills the nematode worm Caenorhabditis elegans. Front Microbiol. 2015;6:1-13.

57. Schöner TA, Gassel S, Osawa A, Tobias NJ, Okuno Y, Sakakibara Y, et al. Aryl polyenes, a highly abundant class of bacterial natural products, are functionally related to antioxidative carotenoids. Chembiochem. 2016;17:247-53

58. Li J-Y, Sugawara F, Harper J, Koshino H, Hess WM, Strobel G. Oocydin a, a chlorinated macrocyclic lactone with potent anti- oomycete activity from Serratia marcescens. Microbiology. 1999;145:3557-64.

59. Thaning C, Welch CJ, Borowicz JJ, Hedman R, Gerhardson B. Suppression of Sclerotinia sclerotiorum apothecial formation by the soil bacterium Serratia plymuthica: identification of a chlorinated macrolide as one of the causal agents. Soil Biol Biochem. 2001;33:1817-26.

60. Matilla MA, Stöckmann H, Leeper FJ, Salmond GPC. Bacterial biosynthetic gene clusters encoding the anti-cancer haterumalide class of molecules: biogenesis of the broad spectrum antifungal and anti-oomycete compound, oocydin a. J Biol Chem. 2012;287:39125-38.

61. Holtsmark I, Eijsink VGH, Brurberg MB. Bacteriocins from plant pathogenic bacteria. FEMS Microbiol Lett. 2008:280:1-7.

62. Grinter R, Milner J, Walker D. Bacteriocins active against plant pathogenic bacteria. Biochem Soc Trans. 2012;40:1498-502.

63. Minowa Y, Araki M, Kanehisa M. Comprehensive analysis of distinctive polyketide and nonribosomal peptide structural motifs encoded in microbial genomes. J Mol Biol. 2007;368:1500-17.

64. Potrykus M, Golanowska M, Hugouvieux-Cotte-Pattat N, Lojkowska E. Regulators involved in Dickeya solani virulence, genetic conservation, and functional variability. Mol Plant-Microbe Interact. 2014;27:700-11.

\section{Ready to submit your research? Choose BMC and benefit from:}

- fast, convenient online submission

- thorough peer review by experienced researchers in your field

- rapid publication on acceptance

- support for research data, including large and complex data types

- gold Open Access which fosters wider collaboration and increased citations

- maximum visibility for your research: over $100 \mathrm{M}$ website views per year

At BMC, research is always in progress.

Learn more biomedcentral.com/submissions 